\title{
Análise de imagens de plântulas para avaliação do potencial fisiológico de sementes de berinjela
}

\author{
Vanessa N Silva ${ }^{1}$; Silvio M Cicero ${ }^{2}$ \\ ${ }^{1}$ Univ. Fed. do Pampa, R. Luiz Joaquim de Sá Britto s/n, Promorar, 97650-000 Itaqui-RS; vnpel@yahoo.com.br; ${ }^{2}$ ESALQ, Av. Pádua \\ Dias 11, 13418-900 Piracicaba-SP; smcicero@usp.br
}

\begin{abstract}
RESUMO
A automatização da análise do crescimento de plântulas pelo uso de processamento computadorizado de imagens pode contribuir para avaliação do vigor de sementes. O objetivo deste trabalho foi verificar a possibilidade de utilização do sistema computadorizado de análise de imagens de plântulas (SVIS, Seed Vigor Imaging System) para detectar diferenças de vigor entre lotes de sementes de berinjela em comparação às informações fornecidas por testes de vigor tradicionalmente utilizados. Dez lotes de sementes de berinjela, cultivar Embu, foram armazenados por 12 meses em sala com ambiente controlado a $20 \pm 1^{\circ} \mathrm{C}$ e $45-50 \%$ de umidade relativa do ar. O teor de água das sementes foi monitorado e o potencial fisiológico avaliado aos 0,6 e 12 meses após o armazenamento, com os testes de germinação, envelhecimento acelerado (tradicional e com solução saturada de sal), condutividade elétrica, emergência de plântulas em substrato e com o sistema de análise computadorizada de imagens de plântulas (SVIS) (comprimento de plântulas, índices de vigor e de crescimento). $\mathrm{O}$ delineamento experimental utilizado foi inteiramente casualizado com 4 repetições. Os dados foram submetidos à análise de variância e comparação de médias por meio do teste de Tukey $(\mathrm{p} \leq 0,05)$. Os parâmetros obtidos na análise computadorizada de plântulas com o software SVIS ${ }^{\circledR}$ (comprimento de plântulas e índices de vigor e de crescimento) são eficientes para avaliar o potencial fisiológico de sementes de berinjela, de forma similar às avaliações rotineiramente utilizadas para essa finalidade.
\end{abstract}

Palavras-chave: Solanum melongena, SVIS, vigor.

\begin{abstract}
Seedling imaging analyze to evaluate eggplant seed physiological potential

The automatization of the analysis of seedling growth by the use of computerized image can contribute to the evaluation of seed vigor. The aim of this research was to verify the efficiency of computerized seedling image analysis by SVIS to detect differences of vigor between eggplant seed lots as compared to those provided by traditional vigor tests. Ten lots of eggplant seeds, cultivar Embu, were stored for 12 months in a room with controlled environment, at $20 \pm 1^{\circ} \mathrm{C}$ and $45-50 \%$ relative humidity. The water content of the seeds was monitored and physiological potential assessed at 0, 6 and 12 months after storage, with standard germination, accelerated aging (traditional and with saturated salt solution), electrical conductivity, seedling emergence on substrate and with the system of computerized image analysis (SVIS) of seedlings (seedling length, vigor and growth index). The experimental design was completely randomized with 4 replications and data were submitted to analysis of variance and means were compared by the Tukey test $(\mathrm{p}<0.05)$. The parameters obtained by the computerized seedling analysis with SVIS ${ }^{\circledR}$ software (seedling length and vigor and growth index) are efficient to differ between lots of high and low vigor eggplant seeds as traditional vigor methods.
\end{abstract}

Keywords: Solanum melongena, SVIS, vigor.

(Recebido para publicação em 22 de fevereiro de 2013; aceito em 25 de março de 2014)

(Received on February 22, 2013; accepted on March 25, 2014)

$\mathrm{P}$ ara obtenção de mudas de hortaliças de qualidade é fundamental o uso de sementes sadias e com elevado potencial fisiológico. A análise desse parâmetro, etapa fundamental para programas de produção, normalmente é realizada por meio do teste de germinação; entretanto como este é conduzido sob condições ideais de temperatura, umidade, luminosidade e aeração, pode superestimar o potencial fisiológico das sementes, sendo adequado complementar a avaliação com uso de um ou mais testes de vigor.

Entre os testes de vigor utilizados comumente, alguns baseiam-se em determinações da velocidade e da uniformidade de crescimento de plântulas ou em avaliações manuais de suas partes, apresentando limitações como a variação de resultados entre laboratórios, por causa da subjetividade da análise e consumo excessivo de tempo para a obtenção dos resultados. A automatização dessas análises pelo uso de processamento computadorizado de imagens pode eliminar as dificuldades citadas (Hoffmaster et al., 2003).

Recentemente, pesquisadores da The Ohio State University nos USA desenvolveram um sistema automatizado para avaliação do vigor de sementes, baseado no crescimento de plântulas, denominado Seed Vigor Imaging System (SVIS) (Sako et al., 2001); para tanto, trabalharam com imagens escaneadas de plântulas de alface, cujas partes eram identificadas e marcadas por software específico. Após o processamento das imagens em computador, obtiveram, simultaneamente, dados referentes ao comprimento da raiz primária, do hipocótilo e da plântula toda, além da relação raiz/hipocótilo e parâmetros baseados na velocidade e uniformidade de crescimento. Este tipo de análise vem possibilitando a determinação do vigor de sementes de outras espécies como milho (Otoni \& McDonald, 2005), melão (Marcos-Filho et al., 2006), soja (Marcos-Filho et al., 2009), crotalária (Silva et al., 2012), pepino (Chiquito et al., 2012), e a avaliação do efeito do condicionamento fisiológico em sementes de milho doce (Gomes Junior et al., 
2009) e Guazuma ulmifolia (Brancalion et al., 2010). Esse método consiste na captação de imagens digitais múltiplas, em escaner, de plântulas, que são processadas em computador, gerando valores numéricos que representam o potencial fisiológico das sementes, com base em parâmetros preestabelecidos. Os dados incluem índices de vigor, de crescimento e comprimento de plântulas (Sako et al., 2001). Os valores do índice de vigor são baseados na rapidez e uniformidade de desenvolvimento das plântulas da amostra, em relação ao máximo valor estimado para plântulas com a idade pré-estabelecida na programação do software. Assim, após o processamento das imagens, o software gera automaticamente valores numéricos referentes ao índice de vigor (valores de 0 a 1000, diretamente proporcionais ao vigor).

Não existem pesquisas até o momento sobre o uso desta técnica para a avaliação do vigor de sementes de berinjela; portanto o objetivo deste trabalho foi verificar a possibilidade de utilização do sistema computadorizado de análise de imagens de plântulas (SVIS, Seed Vigor Image System) para detectar diferenças entre lotes de sementes, em comparação com as informações fornecidas por testes de vigor tradicionalmente utilizados.

\section{MATERIAL E MÉTODOS}

A pesquisa foi conduzida nos laboratórios de Análise de Sementes e de Análise de Imagens da ESALQ, em Piracicaba-SP. Dez lotes de sementes de berinjela, cultivar Embu, tratadas com fungicida Thiram, foram acondicionadas em embalagens de papel aluminizado e armazenadas durante 12 meses em sala com ambiente controlado, a $20 \pm 1^{\circ} \mathrm{C}$ e $45-50 \%$ de umidade relativa do ar e avaliadas por meio dos testes discriminados a seguir.

Teor de água - utilizou-se o método da estufa, a $105 \pm 3^{\circ} \mathrm{C}$, por 24 horas com duas amostras de sementes de 1,0 g para cada lote e os resultados foram expressos em porcentagem (base úmida), conforme Brasil (2009). Germinação - quatro repetições de 50 sementes de cada lote foram distribuídas sobre duas folhas de papel mata-borrão (Germibox) umedecidas com quantidade de água equivalente a 2,5 vezes a massa seca do substrato, no interior de caixas de plástico $(11 \times 11 \times 3,5 \mathrm{~cm})$ e expostas a $20-30^{\circ} \mathrm{C}$, com fotoperíodo ajustado para 8 horas de luz e 16 horas de escuro. As avaliações foram realizadas aos 7 (primeira contagem) e 14 dias após a semeadura (Brasil, 2009). Os resultados foram expressos em porcentagem de plântulas normais para cada lote. Envelhecimento acelerado tradicional (EAT) - uma camada de sementes foi distribuída sobre tela metálica colocada no interior de uma caixa de plástico $(11 \times 11 \times 3,5 \mathrm{~cm})$ contendo $40 \mathrm{~mL}$ de água destilada no fundo. Cada caixa foi tampada e exposta a $41^{\circ} \mathrm{C}$ por 48 horas em incubadora BOD; em seguida foi determinado o teor de água das sementes e realizado teste de germinação, sendo as plântulas avaliadas sete dias após a semeadura (Marcos-Filho, 1999). Envelhecimento acelerado com solução saturada de sal (EASS) - realizado de forma semelhante ao procedimento tradicional, porém utilizando $40 \mathrm{~mL}$ de solução saturada de $\mathrm{NaCl}$ em substituição à água (Jianhua \& McDonald, 1996). Condutividade elétrica - foi utilizada metodologia adaptada de Panobianco \& Marcos Filho (1998), com quatro repetições de 25 sementes de cada lote, as quais foram pesadas e, em seguida, imersas em 25 $\mathrm{mL}$ de água destilada onde permaneceram por 24 horas a $25^{\circ} \mathrm{C}$. A leitura da condutividade elétrica da solução foi realizada em condutivímetro da marca DIGIMED, modelo DM-31. Os valores das leituras foram expressos em $\mu \mathrm{S} / \mathrm{cm} / \mathrm{g}$ de semente. Emergência de plântulas - quatro repetições de 50 sementes por lote foram semeadas em bandejas de isopor contendo substrato comercial próprio para produção de mudas de olerícolas (Plantmax $\left.{ }^{\circledR}\right)$. Aos 14 dias após a semeadura foi realizada a contagem de plântulas emersas (Rodo et al., 1998).

Análise computadorizada de plântulas por meio do software SVIS - para a obtenção de plântulas foram testados preliminarmente os períodos de 3,4 e 5 dias a $20-30^{\circ} \mathrm{C}$ ou $25^{\circ} \mathrm{C}$, em rolos de papel toalha ou sobre papel em caixa de plástico $(11 \times 11 \times 3,5 \mathrm{~cm})$, em presença e ausência de luz. Foi adotada a exposição de 25 sementes a $20-30^{\circ} \mathrm{C}$, em rolos de papel toalha umedecidos com quantidade de água equivalente a 2,5 vezes a massa seca do papel, por 5 dias. Em seguida, as plântulas foram transferidas do papel toalha para uma folha de cartolina com coloração preta e as imagens foram obtidas com escaner modelo HP Scanjet 2004, montado de maneira invertida no interior de uma caixa de alumínio com resolução de 100 dpi. Em seguida, as imagens de plântulas foram analisadas com o uso do software SVIS instalado em computador Pentium IV operado por sistema Windows XP Profissional. Após a análise e a avaliação de cada plântula, o software SVIS forneceu valores referentes aos índices de vigor (IV), de crescimento (IC) e comprimento de plântulas (CP). Os dados de comprimento de plântulas foram fornecidos pelo programa em pixels e, após a tabulação, foram transformados para $\mathrm{cm}$, considerando-se que um pixel corresponde a $0,02645 \mathrm{~cm}$.

O delineamento experimental utilizado foi inteiramente casualizado com quatro repetições. Foram realizadas 3 épocas de avaliação (0, 6 e 12 meses) durante o armazenamento. Os dados foram submetidos à análise de variância e comparação de médias por meio do teste de Tukey $(\mathrm{p} \leq 0,01)$. Além disso, verificou-se o tipo de distribuição dos dados percentuais e aplicou-se o teste de Cochran para verificar se as variâncias apresentavam homogeneidade. Os dados que não apresentaram distribuição normal e homogeneidade de variância foram transformados em $\operatorname{arcsen}(\mathrm{x} / 100)^{1 / 2}$.

\section{RESULTADOS E DISCUSSÃO}

O teor de água das sementes variou entre 6,2 e 7,8\% para os 10 lotes na primeira época de análise (Tabela 1); é importante que este parâmetro seja uniforme entre os lotes, pois diferenças maiores que 2 a 3 pontos percentuais podem interferir nos resultados dos testes de vigor (Marcos-Filho, 1999), já que estes têm influência na velocidade de absorção de água pelas sementes.

$\mathrm{Na}$ caracterização inicial, a ger- 
Tabela 1. Teor de água (TA), germinação (G), primeira contagem de germinação (PC), envelhecimento acelerado tradicional (EAT) e com solução saturada de sal (EASS), emergência de plântulas (EP), condutividade elétrica (CE), índice de vigor (IV), índice de crescimento de plântulas (IC) e comprimento de plântulas (CP) de dez lotes de sementes de berinjela, cultivar Embu, primeira época de avaliação [seed moisture content (TA), germination (G), germination first count (PC), traditional (EAT) and saturated salt accelarated aging (EASS), seedling emergence (EP), electrical conductivity (EC), vigor index (IV), seedling growth index (IC) and seedling lenght (CP) of ten lots of eggplant seeds, cultivar Embu, first evaluation period]. Piracicaba, ESALQ, 2011.

\begin{tabular}{|c|c|c|c|c|c|c|c|c|c|c|}
\hline \multirow{2}{*}{ Lote } & TA & $G^{*}$ & PC* & EAT & EASS* & EP & \multirow{2}{*}{$\begin{array}{c}\mathrm{CE}(\mu \mathrm{S} / \\
\mathrm{cm} / \mathrm{g})\end{array}$} & \multirow{2}{*}{ IV } & \multirow{2}{*}{ IC } & \multirow{2}{*}{$\mathbf{C P}$} \\
\hline & \multicolumn{6}{|c|}{$(\%)$} & & & & \\
\hline 1 & 6,2 & $97 \mathrm{~A}^{1}$ & $84 \mathrm{AB}$ & $85 \mathrm{~A}$ & $80 \mathrm{AB}$ & $85 \mathrm{AB}$ & $244 \mathrm{C}$ & $650 \mathrm{BC}$ & $558 \mathrm{BC}$ & $5,3 \mathrm{~B}$ \\
\hline 2 & 6,3 & $96 \mathrm{~A}$ & $91 \mathrm{~A}$ & $91 \mathrm{~A}$ & $88 \mathrm{AB}$ & $86 \mathrm{AB}$ & $247 \mathrm{BC}$ & $627 \mathrm{BC}$ & $527 \mathrm{C}$ & $4,8 \mathrm{~B}$ \\
\hline 3 & 6,3 & $97 \mathrm{~A}$ & $72 \mathrm{BC}$ & $72 \mathrm{~B}$ & $79 \mathrm{AB}$ & $85 \mathrm{AB}$ & $241 \mathrm{C}$ & $632 \mathrm{BC}$ & $533 \mathrm{C}$ & $4,9 \mathrm{~B}$ \\
\hline 4 & 6,5 & $95 \mathrm{~A}$ & $83 \mathrm{ABC}$ & $83 \mathrm{~A}$ & $76 \mathrm{~B}$ & $84 \mathrm{AB}$ & $254 \mathrm{D}$ & $618 \mathrm{BC}$ & $526 \mathrm{C}$ & $4,6 \mathrm{BC}$ \\
\hline 5 & 6,4 & $97 \mathrm{~A}$ & $91 \mathrm{~A}$ & $91 \mathrm{~A}$ & $85 \mathrm{AB}$ & $94 \mathrm{~A}$ & $187 \mathrm{~A}$ & $737 \mathrm{~A}$ & $677 \mathrm{~A}$ & $6,4 \mathrm{~A}$ \\
\hline 6 & 7,2 & $97 \mathrm{~A}$ & $91 \mathrm{~A}$ & $92 \mathrm{~A}$ & $93 \mathrm{~A}$ & $86 \mathrm{AB}$ & $231 \mathrm{BC}$ & $697 \mathrm{AB}$ & $652 \mathrm{AB}$ & $5,5 \mathrm{AB}$ \\
\hline 7 & 7,7 & $97 \mathrm{~A}$ & $94 \mathrm{~A}$ & $94 \mathrm{~A}$ & $84 \mathrm{AB}$ & $95 \mathrm{~A}$ & $189 \mathrm{~A}$ & $771 \mathrm{~A}$ & $748 \mathrm{~A}$ & $6,5 \mathrm{~A}$ \\
\hline 8 & 7,6 & $95 \mathrm{~A}$ & $72 \mathrm{BC}$ & $72 \mathrm{~B}$ & $48 \mathrm{C}$ & $86 \mathrm{AB}$ & $240 \mathrm{BC}$ & $572 \mathrm{C}$ & $481 \mathrm{CD}$ & $3,6 \mathrm{C}$ \\
\hline 9 & 7,7 & $97 \mathrm{~A}$ & $63 \mathrm{CD}$ & $63 \mathrm{C}$ & $39 \mathrm{C}$ & $89 \mathrm{AB}$ & $249 \mathrm{C}$ & $617 \mathrm{BC}$ & $526 \mathrm{C}$ & $4,5 \mathrm{BC}$ \\
\hline 10 & 7,8 & $87 \mathrm{~B}$ & $49 \mathrm{D}$ & $49 \mathrm{C}$ & $32 \mathrm{C}$ & $73 \mathrm{~B}$ & $214 \mathrm{AB}$ & $440 \mathrm{D}$ & $370 \mathrm{D}$ & $1,6 \mathrm{D}$ \\
\hline CV (\%) & - & 7,4 & 10,4 & 9,5 & 8,7 & 7,9 & 5,4 & 5,4 & 8,7 & 8,7 \\
\hline
\end{tabular}

${ }^{1}$ Médias seguidas de mesma letra na coluna, para cada época experimental, não diferem entre si pelo teste de Tukey ( $\left.\leq 00,05\right) ; *$ Dados transformados em $\operatorname{arcsen}(\mathrm{x} / 100)^{1 / 2}\left[{ }^{1}\right.$ means followed by the same letter do not differ by Tukey test $(\mathrm{p} \leq 0.05) ;{ }^{*}$ data transformed at arcsin $\left.(\mathrm{x} / 100)^{1 / 2}\right]$.

Tabela 2. Teor de água (TA), germinação (G), primeira contagem de germinação (PC), envelhecimento acelerado tradicional (EAT) e com solução saturada de sal (EASS), emergência de plântulas (EP), condutividade elétrica (CE), índice de vigor (IV), índice de crescimento de plântulas (IC) e comprimento de plântulas (CP) de dez lotes de sementes de berinjela, cultivar Embu, segunda época de avaliação [seed moisture content (TA), germination (G), germination first count (PC), traditional (EAT) and saturated salt accelerated aging (EASS), seedling emergence (EP), electrical conductivity (EC), vigor index (IV), seedling growth index (IC) and seedling lenght (CP) of ten lots of eggplant seeds cultivar Embu, second evaluation period]. Piracicaba, ESALQ, 2011.

\begin{tabular}{|c|c|c|c|c|c|c|c|c|c|c|}
\hline \multirow{2}{*}{ Lote } & TA & $G^{*}$ & PC* & EAT & EASS* & EP & \multirow{2}{*}{$\begin{array}{c}\mathrm{CE}(\mu \mathrm{S} / \\
\mathrm{cm} / \mathrm{g})\end{array}$} & \multirow{2}{*}{ IV } & \multirow{2}{*}{ IC } & \multirow{2}{*}{$\mathbf{C P}$} \\
\hline & & & & $(\%)$ & & & & & & \\
\hline 1 & 7,3 & $93 \mathrm{AB}^{1}$ & $90 \mathrm{~A}$ & $44 \mathrm{DEF}$ & $67 \mathrm{AB}$ & $90 \mathrm{AB}$ & $234 \mathrm{D}$ & $687 \mathrm{~B}$ & $602 \mathrm{~B}$ & $5,5 \mathrm{~B}$ \\
\hline 2 & 7,2 & $93 \mathrm{AB}$ & $91 \mathrm{~A}$ & $63 \mathrm{ABC}$ & $81 \mathrm{~A}$ & $76 \mathrm{BC}$ & $219 \mathrm{BC}$ & $657 \mathrm{~B}$ & $573 \mathrm{~B}$ & $4,7 \mathrm{~B}$ \\
\hline 3 & 7,5 & $91 \mathrm{ABC}$ & $87 \mathrm{AB}$ & $60 \mathrm{ABCD}$ & $88 \mathrm{~A}$ & $81 \mathrm{ABC}$ & $215 \mathrm{BC}$ & $618 \mathrm{~B}$ & $521 \mathrm{~B}$ & $4,3 \mathrm{~B}$ \\
\hline 4 & 7,3 & $94 \mathrm{~A}$ & $90 \mathrm{~A}$ & $54 \mathrm{BCDE}$ & $76 \mathrm{~A}$ & $90 \mathrm{AB}$ & $206 \mathrm{C}$ & $636 \mathrm{~B}$ & $540 \mathrm{~B}$ & $4,8 \mathrm{~B}$ \\
\hline 5 & 7,9 & $95 \mathrm{~A}$ & $93 \mathrm{~A}$ & $69 \mathrm{AB}$ & $80 \mathrm{~A}$ & $90 \mathrm{AB}$ & $103 \mathrm{~A}$ & $843 \mathrm{~A}$ & $815 \mathrm{~A}$ & $7,6 \mathrm{~A}$ \\
\hline 6 & 7,8 & $93 \mathrm{AB}$ & $88 \mathrm{AB}$ & $62 \mathrm{ABCD}$ & $83 \mathrm{~A}$ & $91 \mathrm{AB}$ & $103 \mathrm{~A}$ & $806 \mathrm{~A}$ & $775 \mathrm{~A}$ & $6,9 \mathrm{~A}$ \\
\hline 7 & 7,8 & $90 \mathrm{ABC}$ & $85 \mathrm{AB}$ & $78 \mathrm{~A}$ & $85 \mathrm{~A}$ & $94 \mathrm{~A}$ & $107 \mathrm{~A}$ & $815 \mathrm{~A}$ & $779 \mathrm{~A}$ & $7,4 \mathrm{~A}$ \\
\hline 8 & 7,2 & $81 \mathrm{CD}$ & $72 \mathrm{BC}$ & $40 \mathrm{EF}$ & $66 \mathrm{AB}$ & $93 \mathrm{AB}$ & $205 \mathrm{C}$ & $651 \mathrm{~B}$ & $579 \mathrm{~B}$ & $4,5 \mathrm{~B}$ \\
\hline 9 & 7,1 & $85 \mathrm{BCD}$ & $80 \mathrm{ABC}$ & $50 \mathrm{CDEF}$ & $41 \mathrm{~B}$ & $89 \mathrm{AB}$ & $212 \mathrm{C}$ & $681 \mathrm{~B}$ & 599 B & $5,2 \mathrm{~B}$ \\
\hline 10 & 7,4 & $78 \mathrm{D}$ & $63 \mathrm{C}$ & $32 \mathrm{~F}$ & $41 \mathrm{~B}$ & $64 \mathrm{C}$ & $151 \mathrm{~B}$ & $443 \mathrm{C}$ & $391 \mathrm{C}$ & $1,4 \mathrm{C}$ \\
\hline $\mathrm{CV}(\%)$ & - & 5,6 & 7,4 & 14,0 & 11,2 & 8,8 & 4,9 & 4,6 & 6,3 & 10,6 \\
\hline
\end{tabular}

${ }^{1}$ Médias seguidas de mesma letra na coluna, para cada época experimental, não diferem entre si pelo teste de Tukey ( $\left.\mathrm{p} \leq 0,05\right) ;{ }^{*} \mathrm{Dados}$ transformados em $\operatorname{arc} \operatorname{sen}(\mathrm{x} / 100)^{1 / 2}\left[{ }^{1}\right.$ means followed by the same letter do not differ by Tukey test $(\mathrm{p} \leq 0.05) ;{ }^{*}$ data transformed at arcsin $\left.(\mathrm{x} / 100)^{1 / 2}\right]$.

minação para a maioria dos lotes foi superior a 95\% (Tabela 1). O percentual de plântulas normais foi similar entre os lotes, sendo que somente o lote 10 foi inferior aos demais. Por ser conduzido em condições ideais de temperatura, umidade, aeração e luminosidade esta avaliação pode não detectar diferenças sutis entre lotes de sementes. Por outro lado, na segunda e terceira épocas foi possível detectar diferenças entre os lotes, possivelmente devido ao avanço da deterioração.

Os testes de vigor, entretanto, indicaram diferenças entre os lotes desde a caracterização inicial. Na primeira contagem de germinação foi possível verificar diferenças que não foram detectadas entre os dados da porcentagem final de germinação. Lotes de sementes com germinação semelhante frequentemente mostram diferenças em suas velocidades de germinação, indicando que existem diferenças de vigor entre 
eles, o que pode ser avaliado na primeira contagem do teste de germinação (Nakagawa, 1999). Contudo, em cada época de avaliação, o ranqueamento dos lotes ocorreu de forma diferente, sendo que houve melhor desempenho dos lotes 2, 5, 6 e 7 comparado aos lotes 3, 8, 9 e 10 (primeira época), dos lotes 1, 2, 4 e 5 comparado ao 8 e 10 (segunda época) e lote 5 em relação aos demais lotes (terceira época), conforme se verifica nas Tabelas 1, 2 e 3.

Essa diferença de ranqueamento entre os lotes também ocorreu em outros testes utilizados para avaliar o vigor. É importante ressaltar que embora os lotes tenham sido formados homogeneamente, é possível ocorrer diferenças no grau de deterioração das sementes que o compõem, pois sementes de um mesmo lote, e até mesmo partes de uma mesma semente, deterioram com velocidades diferentes (Marcos-Filho, 2005), podendo existir diferenças no potencial fisiológico em consequência disto, o que pode contribuir para este resultado.

No teste de envelhecimento acelerado, os dois procedimentos permitiram a detecção de diferenças de vigor das sementes entre os lotes em todas épocas de análise (Tabelas 1, 2 e 3). De maneira geral, os lotes 5, 6 e 7 foram os mais vigorosos em relação aos vários lotes e os lotes 9 e 10 apresentaram o menor vigor, na maioria das épocas.

Na maioria das situações observadas neste trabalho, houve efeito mais drástico no teste tradicional; deve-se considerar que sementes pequenas absorvem água mais rápida e desuniformemente durante o período de envelhecimento, o que pode acelerar o processo de deterioração ou resultar em comportamento variável entre as sementes de uma amostra (Bhering et al., 2006). O uso de solução saturada de sal permite a absorção de água pelas sementes com menor velocidade e a deterioração seja assim menos intensa; porém, houve casos isolados, como os lotes 5 e 7 que apresentaram maior percentual de germinação após o envelhecimento tradicional. Resultados semelhantes também foram verificados para sementes de berinjela (Torres \& Negreiros, 2008). Na terceira época, entretanto, a germinação foi superior no envelhecimento acelerado tradicional comparado ao teste conduzido com solução saturada de sal; porém, cabe destacar que, nesta época, na maioria dos lotes as sementes já estavam muito deterioradas, atingindo somente $13 \%$ de germinação para alguns lotes, fato que pode ter contribuído para este resultado.
A emergência de plântulas em substrato diferenciou os lotes em todas as épocas de avaliação, sendo que ficou mais evidente o menor vigor do lote 10 , em todas as épocas estudadas. $\mathrm{Na}$ primeira época, o percentual de plântulas emersas foi elevado para a maioria dos lotes, exceto para o lote 10; porém, estes resultados foram inferiores aos do teste de germinação (Tabela 1). Como na avaliação da emergência de plântulas não há controle de temperatura, umidade, luminosidade, como no teste de germinação, podem ter ocorrido condições desfavoráveis para as sementes, $o$ que acarretou diferenças no desempenho dos lotes. De maneira geral, o percentual de plântulas emersas foi superior a $85 \%$ para a maior parte dos lotes, durante todo o período do experimento; houve algumas exceções como, por exemplo, para os lotes 2 e 10 na segunda época, e para o lote 10 na terceira época.

A avaliação do vigor de sementes por meio da condutividade elétrica foi sensível a diferenças entre os lotes em todas as épocas de avaliação e, de modo geral, identificou maior potencial fisiológico dos lotes 5, 6 e 7 ao longo do armazenamento (Tabelas 1, 2 e 3), sendo que esses lotes além de apresentarem menor lixiviação de exsudatos,

Tabela 3. Teor de água (TA), germinação (G), primeira contagem de germinação (PC), envelhecimento acelerado tradicional (EAT) e com solução saturada de sal (EASS), emergência de plântulas (EP), condutividade elétrica (CE), índice de vigor (IV), índice de crescimento de plântulas (IC) e comprimento de plântulas (CP) de dez lotes de sementes de berinjela, cultivar Embu, terceira época de avaliação [seed moisture content (TA), germination $(\mathrm{G})$, germination first count (PC), traditional (EAT) and saturated salt accelerated aging (EASS), seedling emergence (EP), electrical conductivity (EC), vigor index (IV), seedling growth index (IC) and seedling lenght (CP) of ten lots of eggplant seeds cultivar Embu, third evaluation period]. Piracicaba, ESALQ, 2011.

\begin{tabular}{|c|c|c|c|c|c|c|c|c|c|c|}
\hline \multirow{2}{*}{ Lote } & TA & $\mathrm{G}^{*}$ & PC* & EAT & EASS* & EP & \multirow{2}{*}{$\begin{array}{c}\mathrm{CE}(\mu \mathrm{S} / \\
\mathrm{cm} / \mathrm{g})\end{array}$} & \multirow{2}{*}{ IV } & \multirow{2}{*}{ IC } & \multirow{2}{*}{$\mathrm{CP}$} \\
\hline & & & & $(\%)$ & & & & & & \\
\hline 1 & 6,2 & $52 \mathrm{CD}^{1}$ & $47 \mathrm{DE}$ & $23 \mathrm{CD}$ & $14 \mathrm{C}$ & $95 \mathrm{AB}$ & $199 \mathrm{BCD}$ & $640 \mathrm{BC}$ & $323 \mathrm{~B}$ & $3,5 \mathrm{~B}$ \\
\hline 2 & 6,3 & $82 \mathrm{AB}$ & $77 \mathrm{ABC}$ & $25 \mathrm{BCD}$ & $13 \mathrm{C}$ & $93 \mathrm{AB}$ & $211 \mathrm{CD}$ & $602 \mathrm{C}$ & $357 \mathrm{~B}$ & $3,5 \mathrm{~B}$ \\
\hline 3 & 6,2 & $57 \mathrm{CD}$ & $50 \mathrm{DE}$ & $30 \mathrm{ABCD}$ & $18 \mathrm{BC}$ & $90 \mathrm{AB}$ & $195 \mathrm{BCD}$ & $622 \mathrm{C}$ & $307 \mathrm{~B}$ & 2,9 B \\
\hline 4 & 6,0 & $58 \mathrm{C}$ & $52 \mathrm{D}$ & $31 \mathrm{ABCD}$ & $23 \mathrm{ABC}$ & $88 \mathrm{BC}$ & $210 \mathrm{CD}$ & $595 \mathrm{C}$ & $331 \mathrm{~B}$ & $3,4 \mathrm{~B}$ \\
\hline 5 & 7,0 & $89 \mathrm{~A}$ & $88 \mathrm{~A}$ & $40 \mathrm{AB}$ & $32 \mathrm{~A}$ & $97 \mathrm{AB}$ & $112 \mathrm{~A}$ & $788 \mathrm{~A}$ & $570 \mathrm{~A}$ & $5,7 \mathrm{~A}$ \\
\hline 6 & 6,8 & $84 \mathrm{AB}$ & $81 \mathrm{AB}$ & $37 \mathrm{ABC}$ & $35 \mathrm{~A}$ & $95 \mathrm{AB}$ & $152 \mathrm{AB}$ & $764 \mathrm{~A}$ & $605 \mathrm{~A}$ & $6,0 \mathrm{~A}$ \\
\hline 7 & 6,8 & $86 \mathrm{~A}$ & $84 \mathrm{AB}$ & $44 \mathrm{~A}$ & $29 \mathrm{AB}$ & $98 \mathrm{~A}$ & $107 \mathrm{~A}$ & $738 \mathrm{AB}$ & $607 \mathrm{~A}$ & $5,4 \mathrm{~A}$ \\
\hline 8 & 6,6 & $65 \mathrm{BC}$ & $63 \mathrm{BCD}$ & $27 \mathrm{BCD}$ & $16 \mathrm{C}$ & $90 \mathrm{AB}$ & $247 \mathrm{D}$ & $605 \mathrm{C}$ & 397 B & $3,6 \mathrm{~B}$ \\
\hline 9 & 6,4 & $57 \mathrm{CD}$ & $55 \mathrm{CD}$ & $19 \mathrm{D}$ & $17 \mathrm{BC}$ & $90 \mathrm{AB}$ & $205 \mathrm{BCD}$ & $582 \mathrm{C}$ & $377 \mathrm{~B}$ & $3,6 \mathrm{~B}$ \\
\hline 10 & 6,2 & $36 \mathrm{D}$ & $28 \mathrm{E}$ & $17 \mathrm{D}$ & $13 \mathrm{C}$ & $73 \mathrm{C}$ & $183 \mathrm{BC}$ & $393 \mathrm{D}$ & $210 \mathrm{C}$ & $1,3 \mathrm{C}$ \\
\hline CV (\%) & - & 9,9 & 14,5 & 22,7 & 12,7 & 6,9 & 12,4 & 6,6 & 9,4 & 9,9 \\
\hline
\end{tabular}

${ }^{1}$ Médias seguidas de mesma letra na coluna, para cada época experimental, não diferem entre si pelo teste de Tukey (p $\left.\leq 0,05\right) ; *$ Dados transformados em $\operatorname{arc} \operatorname{sen}(\mathrm{x} / 100)^{1 / 2}\left[{ }^{1}\right.$ means followed by the same letter do not differ by Tukey test $(\mathrm{p} \leq 0.05) ;{ }^{*}$ data transformed at arcsin $\left.(\mathrm{x} / 100)^{1 / 2}\right]$. 


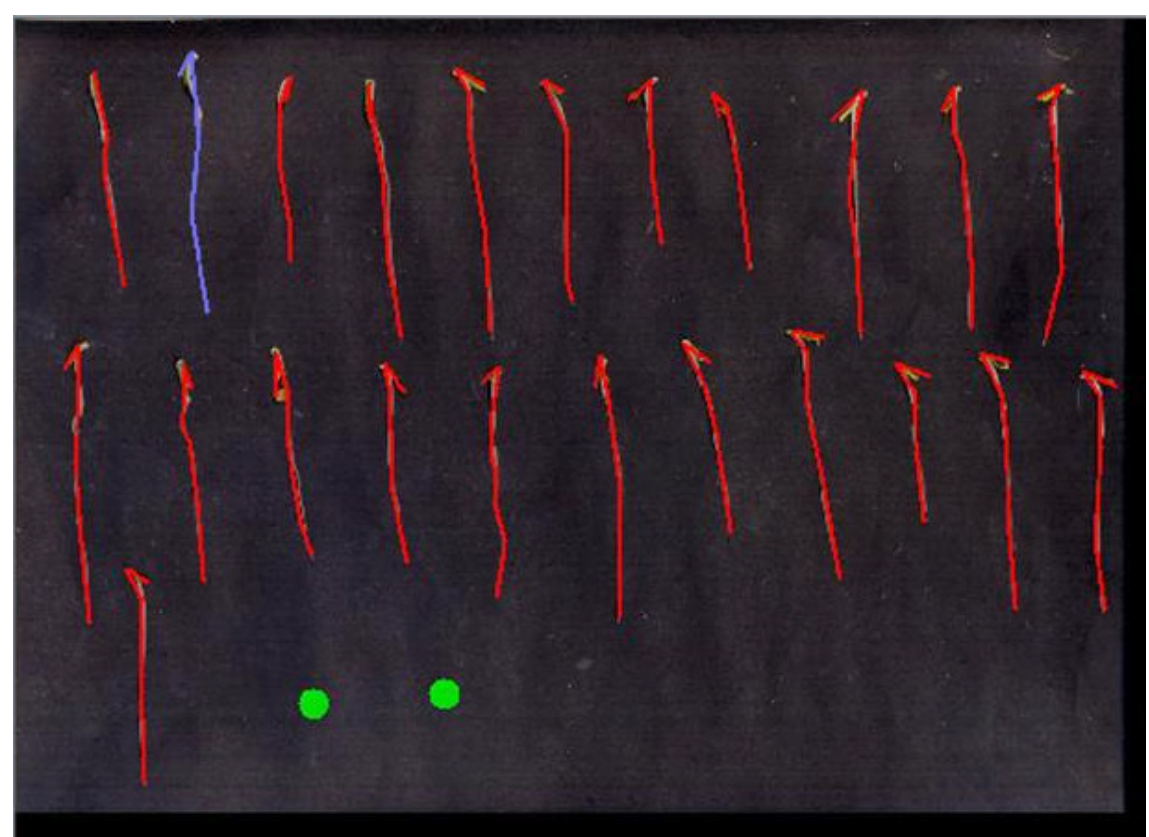

Figura 1. Plântulas de berinjela com 5 dias de idade analisadas no software SVIS. Em destaque comprimento total da plântula marcada em azul e os índices de vigor e crescimento [eggplant seedlings 5-day old analyzed by SVIS software. Example of featured total seedling length (marked in blue) and growth and vigor indexes]. Piracicaba, ESALQ, 2011.

foram os mais vigorosos conforme os dados de emergência de plântulas e primeira contagem de germinação. Provavelmente, a reorganização das membranas celulares das sementes foi mais eficiente que nos demais lotes e, com isso, houve menor perda de íons, resultando em maior eficiência na embebição e reações metabólicas necessárias para o crescimento de plântulas. Deve-se lembrar que a secagem e reidratação da semente impõem considerável estresse sobre os componentes celulares e a lixiviação de solutos durante a embebição indica danos temporários na membrana e organelas como as mitocôndrias, que são vitais para a eficiência da respiração e a liberação de energia (Nonogaki et al., 2010).

Observando o desempenho dos lotes no conjunto de testes utilizados, verificou-se de maneira geral, nas três épocas de armazenamento, superioridade dos lotes 5, 6 e 7 e inferioridade dos lotes 8,9 e 10 (Tabelas 1,2 e 3).

A análise computadorizada de imagens de plântulas pelo SVIS foi capaz de diferenciar os lotes quanto ao vigor em todas as épocas de avaliação, revelando maior potencial fisiológico dos lotes 5, 6 e 7 (Tabelas 1,2 e 3). O índice de razão disto foram mais afetados pelo envelhecimento acelerado. É interessante observar que o lote 9 embora com alto percentual de germinação (97\%), apresentou desempenho deficiente nas demais avaliações de vigor, o que reforça a importância da utilização de vários testes para caracterizar o potencial fisiológico de lotes de sementes.

O uso de parâmetros capazes de detectar diferenças de vigor entre lotes com germinação semelhante é desejável na análise de sementes; além disso, é fundamental que estes sejam coerentes com as avaliações rotineiramente utilizadas, pois apesar de não existirem testes de vigor padronizados para a maioria das espécies cultivadas, há referências na literatura citando o uso de testes como o envelhecimento acelerado (Demir et al., 2005; Torres \& Negreiros, 2008) e deterioração controlada (Demir et al., 2005) para sementes de berinjela.

Utilizando a análise computadorizada de imagens de plântulas por meio do SVIS, outras pesquisas, assim como esta, mostraram coerência dos resultados obtidos no índice de vigor. Silva et al. (2012) verificaram relação positiva entre os resultados do índice de vigor de plântulas de crotalária e do teste de condutividade elétrica. Contreras \& Barros (2005) por sua vez, observaram concordância entre os resultados do índice de vigor, da primeira contagem de germinação e do envelhecimento acelerado para sementes de alface, assim como Peñalosa et al. (2005) constataram em relação ao envelhecimento acelerado com solução saturada de sal e a emergência de plântulas.

Sendo assim, o índice de vigor cumpriu finalidades de uma avaliação dessa natureza, que segundo Bennett (2002) são: maior sensibilidade para avaliar o aspecto fisiológico da qualidade de sementes do que o teste de germinação e fornecer resposta consistente com a classificação dos lotes segundo o seu desempenho, ser objetivo, rápido, simples e econômico, repetível e de fácil interpretação.

Outro índice fornecido pelo SVIS foi o de crescimento de plântulas, que revelou diferenças entre os lotes nas três épocas de avaliação, indicando maior potencial fisiológico dos lotes 5 
e 7, em comparação aos lotes 2, 3, 4, 8, 9 e 10, na primeira época, e dos lotes 5, 6 e 7, em relação aos demais, na segunda e terceira épocas (Tabelas 1, 2 e 3); além de diferenciar os lotes, esse índice forneceu resultados semelhantes aos obtidos no conjunto de avaliações comumente utilizadas para avaliar vigor de sementes, destacando maior potencial dos lotes 5, 6 e 7 nas três épocas de análise. Vários trabalhos de pesquisa utilizando a análise computadorizada relatam sua eficiência para diferentes espécies, como Impatiens wallerana (Oakley et al., 2004), melão (Marcos-Filho et al., 2006), soja (Marcos-Filho et al., 2009), canola (Tohidloo \& Kruse, 2009) e pepino (Chiquito, et al., 2012).

Ainda, o uso do SVIS para a análise computadorizada de imagens de plântulas permite avaliar o potencial fisiológico de sementes por meio de mais um parâmetro, o comprimento de plântulas, que classificou os lotes de maneira semelhante aos demais testes de vigor em todas as épocas (Tabelas 1 , 2 e 3). Um exemplo de análise do comprimento de plântulas executado pelo software SVIS pode ser observado na Figura 1, na qual a plântula marcada em azul foi mensurada, indicando o valor de 313 pixels, que corresponde a $8,3 \mathrm{~cm}$.

O comprimento de plântulas, avaliado manualmente, já se mostrou eficiente na avaliação do vigor de sementes de várias espécies; no entanto, é um procedimento que consome tempo considerável para sua realização e está sujeito a erros de interpretação pela subjetividade inerente ao teste, o que pode ser solucionado pela análise computadorizada, que é rápida e precisa. Consequentemente, a automatização desses procedimentos, via recursos computacionais, pode contribuir significativamente para aprimorar a precisão de resultados, além da obtenção mais rápida da informação (Marcos-Filho et al., 2009).

Deve-se ressaltar que uma vantagem adicional da análise do comprimento de plântulas, via SVIS, é a detecção da principal anormalidade encontrada nas plântulas, o encurtamento das raízes, o que não é possível com o uso de alguns testes de vigor, como por exemplo o teste de emergência de plântulas, o qual é muito utilizado para várias espécies.
É importante mencionar que muitos pesquisadores consideram a avaliação da raiz primária um parâmetro fundamental para determinação do atributo fisiológico da qualidade de sementes.

Os resultados obtidos com a análise computadorizada de imagens de plântulas evidenciam a possibilidade do uso do SVIS para a avaliação segura do potencial fisiológico de sementes de berinjela, pois, de forma geral, a classificação dos lotes em níveis de vigor pelos índices do SVIS foi semelhante àquela obtida nas avaliações comumente utilizadas para avaliação de vigor de sementes nas três épocas experimentais.

Os resultados da presente pesquisa indicam nova possibilidade na área de análise de sementes de hortaliças, como a berinjela, pois o uso da análise computadorizada de plântulas permitiu a avaliação segura e prática do potencial fisiológico das sementes, em curto intervalo de tempo.

Em conclusão, o comprimento de plântulas e os índices de vigor e de crescimento obtidos na análise via SVIS são eficientes para separar os lotes em níveis de vigor de forma similar às avaliações rotineiramente utilizadas para essa finalidade.

\section{AGRADECIMENTOS}

\section{À Fundação de Amparo à Pesquisa} do Estado de São Paulo (FAPESP) pela bolsa de Doutorado concedida à primeira autora e pelo financiamento para realização desta pesquisa (processo 2009/51689-3) e pelos recursos financiados pelo Projeto Temático (processo 06/57900-0).

\section{REFERÊNCIAS}

BENNETT M. 2002. Saturated salt accelerated aging (SSAA) and other vigor tests for vegetable seeds. In: Proceedings international seed seminar: Trade, production and technology. Santiago-Chile: PUC de Chile. p.188-193.

BHERING MC; DIAS DCFS; VIDIGAL DS; NAVEIRADSP. 2006. Teste de envelhecimento acelerado em sementes de pimenta. Revista Brasileira de Sementes 28: 64-71.

BRANCALION PHS; TAY D; NOVEMBRE ADLC; RODRIGUES RR; MARCOS-FILHO J. 2010. Priming of pioneer tree Guazuma ulmifolia (Malvaceae) seeds evaluated by an automated computer image analysis. Scientia Agricola 67: 274-279.

BRASIL. Ministério da Agricultura, Pecuária e Abastecimento. 2009. Regras para análise de sementes. Ministério da Agricultura, Pecuária e Abastecimento. Secretaria de Defesa Agropecuária. Brasília-DF: Mapa/ACS. 395p.

BRAZ MR; ROSSETO CAV. 2009. Estabelecimento de plântulas e desempenho de plantas em resposta ao vigor dos aquênios de girassol. Ciência Rural 39: 1997-2003.

CHIQUITO AA; GOMES JUNIOR FG; MARCOS-FILHO J. 2012. Assessment of physiological potential of cucumber seeds using the software Seedling Vigor Imaging System $^{\circledR}\left(\mathrm{SVIS}^{\circledR}\right)$. Revista Brasileira de Sementes 34: 255-263.

CONTRERAS S; BARROS M. 2005.Vigor tests on lettuce seeds and their correlation with emergence. Ciencia e Investigacion Agraria 32: 3-10.

DEMIR I; ERMIS S; OKÇU G; MATTHEWS S. 2005. Vigor tests for predicting seedling emergence of aubergine (Solanum melongena) seed lots. Seed Science and Technology 33: 481-484.

GOMES JUNIOR FG; MONDO VHV; CICERO SM; McDONALD MB; BENNETT MA. 2009. Evaluation of priming effects on sweet corn by SVIS. Seed Technology 31: 95-100.

HOFFMASTER AL; FUJIMURA K; MCDONALD MB; BENNETT MA. 2003. An automated system for vigour testing threeday-old soybean seedlings. Seed Science and Technology 31: 701-713.

JIANHUA Z; Mc DONALD MB. 1996. The saturated salt accelerated aging test for small seeded crops. Seed Science and Technology 25: 123-131.

MARCOS-FILHOJ. 1999. Teste de envelhecimento acelerado In: KRZYZANOWSKI FC; VIEIRA RD; FRANÇA NETO JB. (eds). Vigor de sementes: conceitos e testes. Londrina: ABRATES. p.1-21.

MARCOS-FILHO J. 2005. Fisiologia de sementes das plantas cultivadas. Piracicaba: ESALQ. $495 \mathrm{p}$.

MARCOS-FILHO J; BENNETT MA; Mc DONALD MB; EVANS AF; GRASSBAUGH EM. 2006. Assessment of melon seed vigour by an automated computer imaging system compared to traditional procedures. Seed Science and Technology 35: 485-497.

MARCOS-FILHO J; KIKUTI ALP; LIMA LB. 2009. Métodos para avaliação do vigor de sementes de soja, incluindo a análise computadorizada de imagens. Revista Brasileira de Sementes 31: 102-112.

NAKAGAWA J. 1999. Testes de vigor baseados no desempenho de plântulas. In: KRZYZANOWSKI FC, VIEIRA RD; FRANÇA NETO JB. Vigor de Sementes: conceitos e testes. Londrina: ABRATES. 218p.

NONOGAKI H; BASSEL GW; BEWLEY JD. 2010. Germination - Still a mystery. Plant Science 179: 574-581.

OAKLEY K; KESTER ST; GENEVE RL. 2004. Computer-aided digital image analysis of seedling size and growth rate for assessing 
seed vigour in Impatiens. Seed Science and Technology 32: 837-845

OTONI RR; Mc DONALD MB. 2005. Moisture and temperature effects on maize and soybean seedlings using the seed vigor imaging system. Seed Technology 27: 243-247.

PANOBIANCO M; MARCOS-FILHO J. 1998. Comparação entre métodos para avaliação da qualidade fisiológica de sementes de pimentão. Revista Brasileira de Sementes 20: 68-72.

PEÑALOZA P; RAMIREZ-ROSALES G; McDONALD MB; BENNETT MA. 2005. Lettuce seed quality evaluation using seed physical attributes, saturated salt accelerated aging and the seed vigor imaging system. Electronic Journal of Biotechnology 8: 299. 307.

RODO AB; TILLMANN MAA; VILLELA FA. 1998. Testes de vigor na avaliação da qualidade fisiológica de sementes de tomate. Revista Brasileira de Sementes 20: 23-28.

SAKO Y; McDONALD MB; FUJIMURA K; EVANS AF; BENNETT MA. 2001. A system for automated seed vigour assessment. Seed Science and Technology 29: 625-636.

SILVA CB; LOPES MM; MARCOS-FILHO J;
VIEIRA RD. 2012. Automated system of seedling image analysis (SVIS) and electrical conductivity to assess sun hemp seed vigor. Revista Brasileira de Sementes 34: 55-60.

TOHIDLOO G; KRUSE M. 2009. Development of an image analysis aided seedling growth test for winter oilseed rape and verification as a vigour test. Seed Science and Technology 37: $98-109$.

TORRES SB; NEGREIROS MZ. 2008. Envelhecimento acelerado em sementes de berinjela. Revista Brasileira de Sementes 30: 209-213. 